\title{
La comunicación online como herramienta para el fortalecimiento de la ECUARUNARI
}

\author{
The Online Communication as a tool for the ECUARUNARI strengthening
}

\author{
Julia Tatiana Carcelén Ordoñez ${ }^{1}$ \\ Luis Fernando Sarango Macas ${ }^{2}$
}

\section{Resumen}

En la historia ecuatoriana los movimientos sociales desde siempre han jugado un papel determinante en la vida política y social del país, sin embargo, su lucha y contribución han quedado invisibilizadas en las agendas de los medios de comunicación comerciales, violentando así el derecho constitucional de los pueblos originarios a una comunicación legítimamente incluyente. De allí, que este estudio tenga como propósito evidenciar el estado actual de la comunicación que se articula desde la Confederación de Pueblos de la Nacionalidad Kichwa del Ecuador - ECUARUNARI, como pilar fundamental del movimiento indígena ecuatoriano, en aras de fortalecer desde iniciativas propias, procesos de comunicación interculturales. Los resultados del estudio tienen como fuente importante un diagnóstico que profundizó en la manera que se configura la comunicación institucional e intervienen los públicos internos y externos, como parte constitutiva de una estrategia que sostiene el derecho que tienen los movimientos sociales del Ecuador a ejercer la comunicación en condiciones de igualdad y horizontalidad.

Palabras clave: Movimiento indígena; organizaciones sociales; comunicación intercultural; comunicación alternativa; contrainformación; interculturalidad.

\section{Abstract}

In the Ecuadorian history, social movements have always played a decisive role in the political and social life of the country, however, their struggle and contribution have been made invisible in the agendas of the commercial media, thus violating the constitutional right of the indigenous peoples to a legitimately inclusive communication. Hence, this study aims to highlight the current state of communication articulated by the Confederation of Kichwa Nationality Peoples from Ecuador-ECUARUNARI, as a fundamental pillar of the Ecuadorian indigenous movement, in order to strengthen its own initiatives, processes of intercultural communication. The results of the study have as an important source a diagnosis that deepened in the way that institutional

1 Máster en Comunicación Intercultural con Enfoque en Género, Lcda.en Comunicación Social, Periodista Digital y Willachik de la Pluriversidad Amawtay Wasi del Ecuador. Email: tatianacarcelen@gmail.com - ORCID: https://orcid.org/oooo-0003- 0328-6967

2 Estudiante Doctoral y Máster en Docencia Universitaria. Pusha/Rector de la Pluriversidad Amawtay Wasi del Ecuador. Email: pushak1@yahoo.com 
communication is configured and internal and external audiences intervene, as a constitutive part of a strategy that sustains the right that Ecuador's social movements have to perform communication in conditions of equality and horizontality.

Keywords: Indigenous movement; social organizations; intercultural communication; alternative communication; contrainformation; interculturality.

\section{Introducción}

En Ecuador en el año 2008 se aprobó mediante referéndum, la Constitución Política más garantista de la historia del país, en ella se lograron reconocer derechos para los pueblos originarios incluidos, educación y comunicación intercultural. Sin embargo, el escenario político imperante ha influido en que no se les permita acceder en forma plena a los pueblos originarios a su derecho a ejercer una comunicación propia.

Por distintos intereses este derecho constitucional se ha mantenido en "letra muerta", pues los temas concernientes al movimiento no son incluidos en las agendas de los medios de comunicación comerciales. De allí la importancia y necesidad de establecer alternativas comunicacionales que viabilicen y visibilicen estas demandas, mediante un trabajo articulado que fortalezca estos procesos.

En calidad de periodista con trayectoria de trabajo profesional muy cercano a las organizaciones sociales, mi compromiso ha sido promover una comunicación al servicio de los sectores sociales, fortalecer la minka (trabajo conjunto en bien de la comunidad), defender la madre tierra y los derechos de los pueblos originarios. Con estos criterios, surge la necesidad de documentar y sistematizar las cosmovisiónes, los conocimientos ancestrales y la experiencia comunicacional de la Organización Kichwa ECUARUNARI. Para esto, se propuso recuperar en un ambiente participativo las distintas posturas de la Organización respecto a los aciertos, desaciertos y desafios de las estrategias comunicacionales en aras de trazar planes que permitan construir realidades que contribuyan a caminar la palabra, el diálogo y el fortalecimiento de lazos entre las organizaciones.

Tal como lo sostiene Félix Cadena

"Un proceso intencionado de creación participativa de conocimientos teóricos y prácticos, desde y acerca de las prácticas de transformación emancipadora, con el propósito de que ésta pueda de mejor manera lograr sus finalidades de contribuir al desarrollo creciente de la fuerza y de las capacidades de los pueblos y sectores populares para que, conformándose como sujetos colectivos, puedan ser verdaderos protagonistas en la identificación y resolución de sus necesidades y anhelos, tanto 
cotidianos como históricos, superando las relaciones y mecanismos de subordinación que se oponen a esta" (HEGOA, 2004:14).

Frente a la pasividad y poca voluntad política de asegurar espacios en los medios de comunicación a los pueblos originarios se hace impostergable establecer alternativas comunicacionales que integren su dinámica y planteamientos. Por consiguiente, se propone fortalecer las iniciativas comunicacionales de ECUARUNARI procediendo a realizar un diagnóstico en la Central de la Organización ubicada en la ciudad de Quito, para determinar las condiciones en que funcionan.

\section{Revisión de literatura}

Desde sus inicios la comunicación ha desempeñado un rol importante para el desarrollo del ser humano y de las sociedades, con el tiempo y acorde al crecimiento de las necesidades comunicacionales éstas formas de comunicación han ido evolucionando desde las señas, pictogramas, sonidos guturales, letras, palabras, imagen estática, hasta la combinación de audio con imagen en movimiento acompañado de herramientas.

Para Francois (1977): "La comunicación es la transferencia de la información por medio de mensajes. Un mensaje es una sustancia que ha recibido cierta forma; por ejemplo, las vibraciones acústicas del mensaje oral, los impulsos eléctricos del mensaje telefónico, las formas visuales del mensaje escrito, el surco grabado del disco fonográfico, etc."

Y es esa comunicación, la que nace de una necesidad natural de 'compartir' la que nos ha permitido establecer lazos entre las sociedades y naciones entendidas como "la comunidad de los ciudadanos de un estado, viviendo bajo el mismo régimen o gobierno y teniendo una comunión de intereses; la colectividad de los habitantes de un territorio con tradiciones, aspiraciones e intereses comunes, y subordinados a un poder central que se encarga de mantener la unidad del grupo el pueblo de un estado, excluyendo el poder gobernante". (Hobsbawn:1992:25-26).

Ecuador es un país muy rico en diversidad cultural y étnica, las costumbres, tradiciones y lengua característicos de cada uno de los 16 pueblos indígenas, sumado a los grupos Afro y mestizos, hacen surgir un espacio importante para el intercambio de experiencias, es ahí donde nace la necesidad de fortalecer organizaciones como la Confederación de Pueblos de la Nacionalidad Kichwa del Ecuador - ECUARUNARI en el aspecto comunicacional, para que de esta manera se puedan crear lazos con otras organizaciones y pueblos como también fortalecer los lazos existentes dentro y fuera de la confederación.

La realización de la tesis "Fortalecimiento de la Comunicación Intercultural en la Confederación de Pueblos de la Nacionalidad Kichwa del Ecuador- ECUARUNARI”, 
de la cual se desprende este artículo, surgió de esa necesidad de afianzar relaciones, compartir historias, sueños y caminos de luchas aunados en una sola voz.

En este orden la categoría de Periodismo Comunitario es una pieza clave para determinar a cual parte de la población se sirve, a la minoría o a la mayoría de esta. Siguiendo a Tabares, Cuando hablamos de este último, estamos hablando de conspiración (respirar juntos) con las comunidades oprimidas, de estar junto a ellas en sus más secretos sueños de amor. En definitiva, el periodismo comunitario, es el que conspira, camina junto, trasciende y hace visible al oprimido no como el marginal, sino como el pobre, real y capaz de superar su condición (Tavares:1998:60).

Por su parte, la Comunicación Alternativa de acuerdo a es la que "surge de la necesidad de los individuos de comentar acerca de su entorno, y exponer su visión del mundo, muchas veces contradictoria a la visión del sistema hegemónico. Esta comunicación se da en particular en sistemas dominantes, en los que los individuos no cuentan con una expresión abierta dentro de los medios y canales establecidos, ya que estos son invadidos, saturados y controlados por el discurso del poder". (Corrales y Hernández, 2009).

Del planteamiento anterior se puede decir, que la Ecuarunari, al igual que muchas organizaciones sociales y sindicatos han sido víctimas de violencia política y un constante linchamiento mediático por parte del régimen por no coincidir políticamente con el partido oficialista, por lo que necesitan de un medio alternativo que comparta su versión de los hechos y aporte al fortalecimiento de relaciones interculturales y de diálogo.

Para Corrales y Moctezuma (2011:11), la interculturalidad tiene el ingrediente político que es el que permite establecer relaciones dialógicas entre culturas, con con otras formas de organización social, y con mecanismos distintos en la toma de decisiones. Es una interculturalidad cuya dinámica social aboga porque no existan condiciones de desigualdad estructural.

Con esta mirada, este artículo se centra en las prácticas de respeto y de diálogo que como Organización se han venido implementando en una búsqueda por asegurar que "las otras voces, las nuestras", también sean incorporadas en la agenda de los medios.

Dado que los recursos económicos son la principal dificultad para sostener las pocas iniciativas comunicacionales, se trabajó en una estrategia para el uso de herramientas que estén al alcance de la Confederación, de fácil manejo y sobre todo que sirvan para la difusión de actividades y propuestas. 


\section{Materiales y métodos}

El estudio compartido, fue guiado metodológicamente por el Cultivo y Crianza de Saberes y Conocimientos, el CCRISAC. Este responde a una nueva metodología de investigación que rompe los esquemas tradicionales y se enfoca en una construcción de saberes y conocimientos a partir de las experiencias, en un ambiente de respeto.

El estudio es de carácter cualitativo, apoyado en el diálogo y testimonios que hicieron posible recoger y plasmar las experiencias vividas por los y las protagonistas tal como lo describen Pita y Pértegas (2002): "la investigación cualitativa trata de identificar la naturaleza profunda de las realidades, su sistema de relaciones, su estructura dinámica (...)".

De esta manera, en este caminar, se determinó la importancia de cumplir con el principio de Interculturalidad entendido como: “(...) el proceso de fortalecimiento interno de las manifestaciones culturales e identitarias de los pueblos, como punto de partida para la generación de diálogos interculturales en igualdad de condiciones, oportunidades y derechos. En el CCRISAC, este principio incide en el diálogo intercultural entre pueblos con identidad cultural, propiciando en este proceso el uso y análisis desde las lenguas y lenguajes propios. Su aplicación es evidente en el fortalecimiento y revitalización de las sabidurías y conocimientos, desde la visión integral de cada pueblo". (RUIICAY, 2015:6)

A partir de este principio trazado por la Red de Universidades Indígenas Interculturales y Comunitarias de Abya Yala (RUIICAY), se implementó el camino vivencial simbólico relacional que busca principalmente recrear y sistematizar los saberes individuales y colectivos para aplicarlos como soluciones a los problemas individuales y colectivos y lograr una vida plena (RUIICAY, 2015: 11).

Los cuatro momentos son resumidos en el siguiente cuadro:

Tabla 1: Momentos Método Vivencial

\begin{tabular}{|c|l|}
\hline $\begin{array}{c}\text { a) Vivenciando y } \\
\text { experienciando }\end{array}$ & $\begin{array}{l}\text { En esta etapa del proceso se realizó un acercamiento más interno a la } \\
\text { ECUARUNARI, para pedir permiso de realizar el estudio y conocer más su propio } \\
\text { funcionamiento. }\end{array}$ \\
\hline $\begin{array}{c}\text { b) Recuperandoy } \\
\text { reencantando }\end{array}$ & $\begin{array}{l}\text { Con el proceso de recopilación de la información y saberes con los miembros del } \\
\text { Consejo de Gobierno se determinaron principales dificultades y posibles solucio- } \\
\text { nes a los procesos comunicacionales. }\end{array}$ \\
\hline $\begin{array}{c}\text { c) Significandoy } \\
\text { resignificando }\end{array}$ & $\begin{array}{l}\text { La información sistematizada evidencia que la principal dificultad que influye en } \\
\text { que las iniciativas se trunquen, es el factor económico. Por tanto, se plantea a la } \\
\text { Organización a manera de alternativa, reforzar el uso de las plataformas digitales } \\
\text { para la comunicación. }\end{array}$ \\
\hline
\end{tabular}


Para cumplir con las etapas de este proceso se implementaron conversatorios, coherente con "el acto de saber conversar, dialogar con él (la) y los(as) otros de manera comunitaria y familiar como un proceso de construir aprendizajes de manera participativa, orientados a resolver problemas individuales y colectivos, a establecer acuerdos y compromisos. (RUIICAY, 2015).

Los conversatorios se realizaron como una especie de canal de transmisión de conocimientos y sabidurías de manera intergeneracional por medio de la palabra. Esta estrategia permitió contar con información ampliada sobre el tema. Otro recurso importante fueron las entrevistas con personas claves y cuyos testimonios fueron acompañados por la documentación institucional y bibliográfica también revisada para obtener mayores insumos.

Desde la sabiduría de los pueblos indígenas, cuando se va a cultivar “ ... hay que hacerlo de corazón, es decir, si no se hace el trabajo sintiendo, los productos de las cosechas no son deliciosas, pero si se hace el trabajo del cultivo sentipensando desde el corazón, los productos de las cosechas serán deliciosas y abundantes, a más de convertirse en escenarios de aprehensión de saberes y conocimientos". (RUIICAY, 2014:16)

Lo anterior conlleva a enfocar la crianza de saberes de forma adecuada, mediante la participación conjunta con los protagonistas, para que dicho proceso cumpla su papel creador e incentivador y contribuya al desarrollo de los distintos procesos, respondiendo a los intereses de la comunidad.

\section{Etapas de la investigación:}

Dicha crianza de saberes se la desarrolló en cuatro etapas:

- Recolección de la información.

- Procesamiento y sistematización de la información.

- Planteamiento de una estrategia para el manejo (de redes oficiales) y la creación de nuevos canales digitales de la ECUARUNARI.

- Socialización de la investigación y la propuesta al Consejo de Gobierno de la Ecuarunari. 


\section{Resultados y discusión}

El movimiento indígena ecuatoriano cuenta con una larga trayectoria en los diferentes procesos de lucha en defensa de su identidad, sus derechos y los de la Madre Tierra. Antes de la década de los setenta eran considerados analfabetos y no podían ejercer el voto universal hasta 1979, a partir de ahí empezaron a organizarse para defenderse en unidad.

De esta manera y bajo la dinámica comunitaria (principio fundamental del movimiento indígena) surgen organizaciones como la Federación Ecuatoriana de Indios (FEI), que es la originaria de la Ecuador Runacunapak Rikcharimui - Confederación de los Pueblos de Nacionalidad Kichwa del Ecuador- ECUARUNARI en 1972 . (ECUARUNARI, 1981).

En 1980 se crea el Consejo Nacional de Coordinación de las Nacionalidades Indígenas del Ecuador (CONACNIE). En 1982 nace la Confederación de Nacionalidades Indígenas de la Amazonía Ecuatoriana (CONFENAIE), las mismas que se convierten en los pilares fundamentales para la fundación en 1986 de la Confederación de Nacionalidades Indígenas del Ecuador (CONAIE), que agrupa a las otras organizaciones que son de carácter regional, constituyéndose así en la principal.

Conforme van pasando los años de lucha van afirmándose en su posición y principios llegando así a cuestionar la estructura estatal y sus diferentes políticas indolentes, ahí empieza una nueva etapa de lucha por reivindicaciones de la sociedad en general.

"la práctica del diálogo hacia la consecución de los consensos, la construcción de un Estado Plurinacional, el reencuentro en la interculturalidad y la diversidad social y cultural, la ampliación y profundización de la democracia, la equidad y la justicia social". (Macas, 2002: 3)

Lamentablemente a pesar de tener una larga trayectoria aún continúan siendo discriminados en el aspecto político, social económico y comunicacional bajo ciertos estereotipos.

"Cuando se piensa en indígenas existe una fuerte tendencia de ver gente con un vestido que podríamos calificar de "tradicional", que hablan una lengua que muchos otros no entienden y que tienen una estrecha relación con el mundo rural, con el mundo campesino. Se piensa que son comunitarios y sobre todo exóticos respecto a la población que habita en la ciudad de Quito, en donde es frecuente ver a algunos de ellos mendigando en la calles. El estereotipo dominante los asocia con la ruralidad, pero además los asocia con los valores más bajos de la escala social, recreando elementos de dominación de unos grupos sobre otros" (Gómez, 2008:108) 
En materia de estereotipos quiero mencionar que esta fue otra de las luchas ancabezadas por el Movimiento en una búsqueda por desestructurar y romper con un sistema marcado por prejuicios y estereotipos, hasta lograr mejores condiciones de vida para los ecuatorianos. Fue así, que vimos trascender el movimiento en los 90 con el Primer Levantamiento Indígena.

Hoy en día el país entero sabe de la existencia de la CONAIE y de sus organizaciones, de las cuales se le considera como el pilar fundamental a la ECUARUNARI, pues es la Confederación que agrupa la mayor cantidad de pueblos indígenas del Ecuador y en la que se centró el trabajo de investigación para ayudar en su fortalecimiento Comunicacional.

Cabe mencionar que existen otro tipo de organizaciones indígenas que no son trascendentales puesto que no desarrollan acciones a nivel nacional como son la Federación Ecuatoriana de Indígenas Evangélicos (FEINE) y, la Federación Nacional de Organizaciones Campesinas, Indígenas y Negras (FENOCIN).

\section{Iniciativas de comunicación impulsadas por la ECUARUNARI}

Luego de un diagnóstico en el que pudo documentarse las distintas estrategias comunicacionales realizadas a lo largo de la vida organizativa de ECUARUNARI, como también la duración que tuvieron y lo que causó su suspensión.

Entre las principales iniciativas comunicacionales impulsadas por dicha organización se encuentran:

Periódico RIKCHARISHUN (Despertémonos), medio de difusión más antiguo de la organización, nació en el año 1983, su principal objetivo es dar a conocer los acontecimientos, reclamos, acciones y propuestas del ECUARUNARI, sus organizaciones, pueblos y comunidades, en el idioma propio. Lamentablemente la falta de recursos económicos impidieron que se imprima el periódico.

Coordinadora de Radios Comunitarias Era una alianza de radios para la transmisión de noticieros, los cuales eran elaborados desde un cabina de la ECUARUNARI en la ciudad de Quito y se los transmitía vía telefónica en horarios específicos y duró tres años.

Red de Comunicadores Interculturales: la regional ECUARUNARI se emprendió en Coordinación con la CONAIE la Red de Comunicadores Interculturales del Ecuador (2010). cuya finalidad era capacitar a los dirigentes provinciales de comunicación.

Uso de las TIC como alternativa: Ante la imperante necesidad de transmitir notas y opiniones importantes y coyunturales que no pueden esperar a un boletín 
impreso, la organización paulatinamente se ha ido sumergiendo en el mundo digital para continuar y ampliar la difusión de sus actividades, principalmente en los canales de facebook, twitter y la web (que en ocasiones se encuentra off line por falta de recursos). "Ha sido una experiencia que ha ido de menos a más, utilizar la tecnología para poder mantener viva a la ECUARUNARI con todas sus actividades de carácter comunicativo; cuando ha habido recursos hemos tenido equipo de gente para trabajar, pero cuando no ha habido como en este caso, me ha tocado hacer ese esfuerzo, hasta el momento". (ZHINGRI, 2016, comunicador de la ECUARUNARI, entrevista realizada en marzo del 2016.)

$Y$ es que el internet se ha convertido en una herramienta muy importante para la organización, pues ante un gobierno que acapara los medios y sus agendas para dedicar espacios prolongados para minimizar, insultar e incluso difamar a todo aquel que no se encuentre dentro de su linea política, por lo cual ECUARUNARI se ha visto en la necesidad de ir implementando cada día más los diferentes espacios que le permitan realizar una contrainformación, en la cual rinden su versión de los acontecimientos, dan pronunciamientos y muestran la verdad oculta al mundo, mediante una estrategia digital que permita, ejecutar de mejor manera la comunicación de la organización mediante el planteamiento de nuevas iniciativas y del fortalecimiento de las ya existentes.

\begin{abstract}
"De nada vale el mejor plan de comunicación si una organización no basa sus acciones en intervenciones sociales concretas. La comunicación ayuda al dialogo, transforma situaciones sociales pero no es milagrosa mi mágica. Como comunicadores somos gestores, articulares y sobre todo productores de sentido en el espacio público. Porque la especificidad de la comunicación institucional para las Organizaciones de la sociedad civil radica en los procesos transformadores y de cambio social que producen" (Amado, 2011:142)
\end{abstract}

Estos son aspectos que llevan a la Organización a considerar que la comunicación es un recurso estratégico para el desarrollo y unidad de los sectores sociales. En este sentido, el Consejo de Gobierno (la ECUARUNARI) destaca la importancia de la misma y ha declarado a la comunicación como una de las prioridades.

"Para nosotros es vital la comunicación, porque primero ayuda a tener un contacto con el tema de la realidad en este caso de las comunidades; luego ayuda a visibilizar las problemáticas pero también las soluciones que se quieren plantear. Entonces la comunicación es como un eje transversal que está en todo y eso es fundamental, sigo insistiendo que en eso tenemos una falencia y ojala en este periodo podamos lograr fortalecerla". (Sánchez, 2016). 
Para fortalecer el proceso comunicacional de ECUARUNARI se procedió a realizar un proceso que consiguió determinar cómo se encuentra la gestión de dichos espacios disponibles en la red, sometiéndolos a una auditoria de redes sociales las cuales se encuentran resumidas en el siguiente FODA.

Tabla 2: FODA Redes Sociales

\begin{tabular}{|c|c|}
\hline FORTALEZAS & OPORTUNIDADES \\
\hline $\begin{array}{l}\text { - Actividades realizadas con las comunidades } \\
\text { - Disponibilidad de material fotográfico y } \\
\text { - Audiovisual para difundir. } \\
\text { - Apoyo de la dirigencia. }\end{array}$ & $\begin{array}{l}\text { - Nuevos proyectos. } \\
\text { - Actividades diarias con las comunidades. } \\
\text { - Alianzas e intercambios con otras organizacio- } \\
\text { nes } \\
\text { - Herramientas y espacios gratuitos en el inter- } \\
\text { net. }\end{array}$ \\
\hline DEBILIDADES & AMENAZAS \\
\hline $\begin{array}{l}\text { - Interacción nula con los seguidores de los } \\
\text { espacios en redes sociales. } \\
\text { - Un solo encargado de comunicación } \\
\text { - Falta de recursos económicos }\end{array}$ & $\begin{array}{l}\text { - Poca visibilidad en las cuentas de Facebook y } \\
\text { Twitter. } \\
\text { - Inactividad. }\end{array}$ \\
\hline
\end{tabular}

En la parte referente a posicionamiento en buscadores se desarrolló un análisis sobre intereses y criterios de búsqueda de los internautas, el cual mostró que los mayores picos de interés de búsqueda del público externo tiene que ver con coyunturas políticas puntuales, donde la Ecuarunari ha sido protagonista como por ejemplo en Agosto del 2015 cuando se desarrolló la Marcha por la Vida y el Levantamiento Indígena, de ahí la actividad ha bajado paulatinamente.

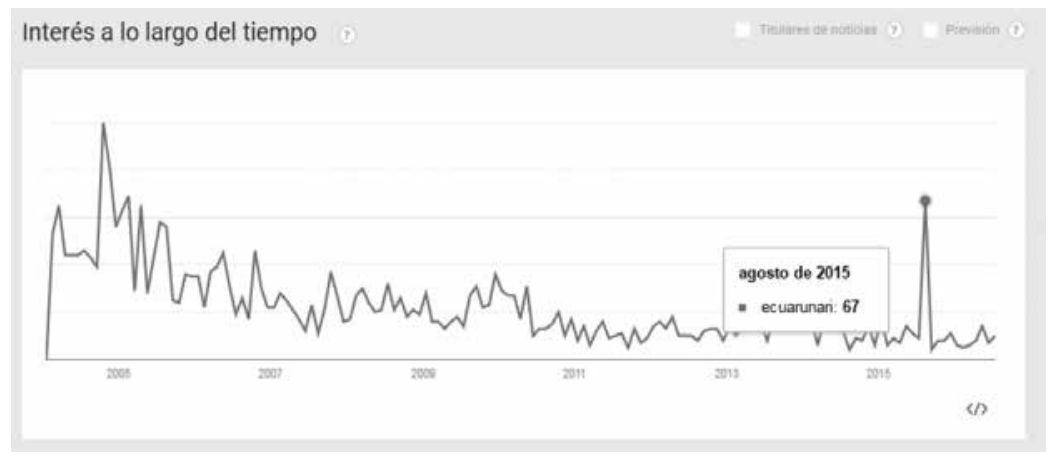

Figura 1: Interés a lo largo del tiempo

Otro aspecto que es importante tomar en cuenta es que los intereses de búsqueda en la red en base a este tema se encuentran únicamente (en las métricas que muestran 
en base al número de búsquedas) concentrados geográficamente en el Ecuador, lo cual dejó ver la necesidad de ampliar los horizontes de difusión y reforzarlos.

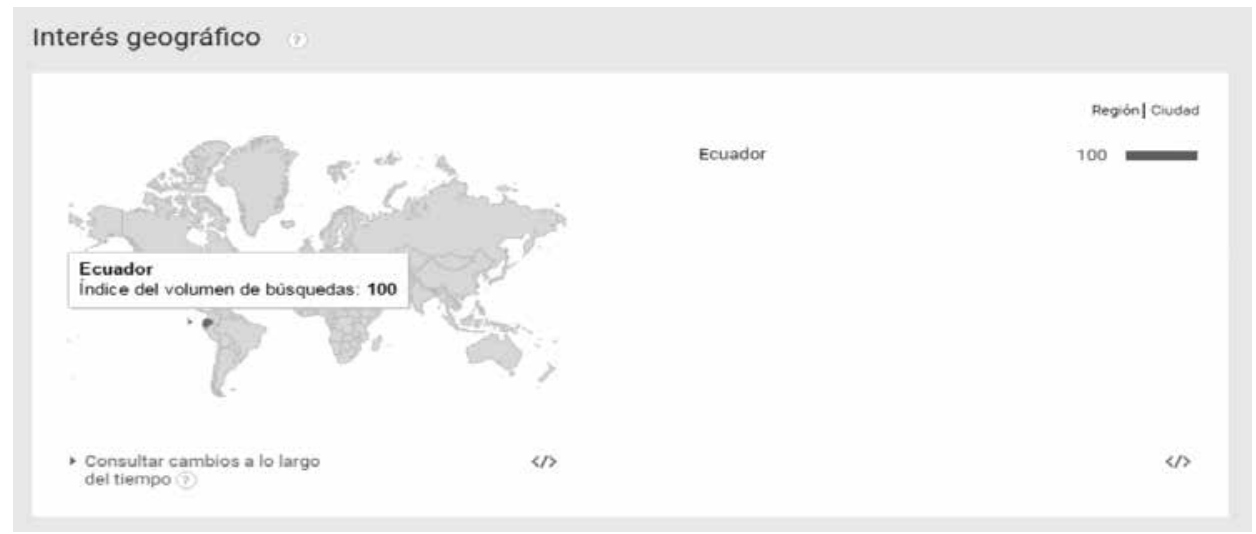

Figura 2: Interés Geográfico

Al ser la comunicación un recurso estratégico que le permite a la organización darse a conocer, proyectarse, ampliar y fortalecer sus relaciones con otras organizaciones, se hace necesario canalizar de mejor manera las fortalezas y oportunidades en favor de la organización, para ir desplazando a las amenazas. Por otra parte luego de la recopilación de la información bibliográfica y consulta a fuentes vivas (antiguos y actuales dirigentes) en cómo se ha articulado la comunicación en los Consejos de Gobierno (éxitos, dificultades, falencias) arrojó como resultado la siguiente problemática en el área de comunicación:

Tabla 3: FODA Comunicacional ECUARUNARI

\begin{tabular}{|c|c|}
\hline FORTALEZAS & OPORTUNIDADES \\
\hline $\begin{array}{l}\text { - Acceso a las comunidades } \\
\text { - Apoyo de la dirigencia. } \\
\text {-La predisposición del dirigente encargado del } \\
\text { área comunicacional para realizar el trabajo } \\
\text {-Apertura organizativa para el desarrollo de la } \\
\text { comunicación }\end{array}$ & $\begin{array}{l}\text { - Nuevos proyectos. } \\
\text { - Actividades con las comunidades. } \\
\text { - Alianzas e intercambios con otras organizaciones } \\
\text { - Herramientas y espacios gratuitos en el internet. } \\
\text { - Acuerdos con los comunicadores de las filiales. }\end{array}$ \\
\hline DEBILIDADES & AMENAZAS \\
\hline $\begin{array}{l}\text { - Un solo encargado de Comunicación } \\
\text { - Falta de recursos humanos } \\
\text { - Falta de recursos económicos } \\
\text { - Cambio de dirigentes de Comunicación } \\
\text { - Poco empoderamiento } \\
\text { - No cuentan con medios propios }\end{array}$ & $\begin{array}{l}\text { - Poca visibilidad en las cuentas de Facebook y } \\
\text { Twitter. } \\
\text { - Poca difusión a causa de los mails perdidos con la } \\
\text { página web. } \\
\text { - Inactividad. } \\
\text { - Inexistencia de una estrategia específica de } \\
\text { comunicación. }\end{array}$ \\
\hline
\end{tabular}


El estudio evidencia como dificultades a las que se suman factores externos, la exclusión de las agendas mediáticas en la prensa comercial, y el constante ataque y desprestigio por parte de sectores "hegemónicos y poderosos", dificultad que en muchas ocasiones les cancela toda oportunidad de brindar su propia versión de los hechos, en flagrante violación de la Constitución en su Art. 66 Numeral 7.

"La comunicación ha sufrido restricciones graves durante este gobierno, que pretende implantar un solo pensamiento y eso se ha podido evidenciar con la expedición de la ley de comunicación, obviamente con esto amenaza persigue amedrenta, incluso nosotros como ECUARUNARI hemos sufrido alguna vez el hackeo de la cuenta de Facebook, eso es todo porque disentimos de la política gubernamental, entonces la comunicación este momento no es tanto un derecho sino que se ha convertido en un privilegio de pocos.

"Por un lado el gobierno a través de sus medios de comunicación insultan, persiguen, estigmatizan a los dirigentes, tachándonos de irracionales, ignorantes, ridículos, indios roscas y toda esa forma peyorativa incluso, nos ha dicho groserías muy fuertes en kichwa. Por otro lado nos queda todo un espacio de debate, de discusión, de participación allá en los grandes medios de comunicación radial, escrita y de televisión del gobierno en los cuales casi no tenemos cabida y no se nos permite expresar nuestros criterios". (Pérez, 2016).

Tras el diagnóstico se tomaron acciones enfocadas en aprovechar recursos online, definido en un proceso comunicacional en Social Media, mediante la optimización de canales, necesarios para poner a disposición el material obtenido de los eventos y demás actividades en las comunidades.

Al establecer lineamientos para el manejo de contenidos en la red, se busca generar el empoderamiento de las bases de la organización ECUARUNARI, y seguir defendiendo sus principios fundamentales por los que ha sido constantemente asediada y linchada mediáticamente. Frente a esta compleja realidad se hizo necesario crear una especie de contrainformación, aprovechando varios recursos gratuitos en la red, de manera que se pudiese fortalecer la comunicación y la identidad institucional y lidiar con las barreras económicas, consideradas por la Organización el "talón de Aquiles".

Obviamente, este es un plan que requiere incorporar la interculturalidad como la base para promover la colectividad y una comunicación estrechamente ligada a los principios fundamentales de la organización, la generación de criterios, el reconocimiento a la diversidad cultural y la construcción de saberes en un ambiente de respeto a la identidad propia. 
"Los comunicadores, al ser especialistas en elaborar mensajes, abrir espacios de diálogo y construir sentido y significados, tienen la habilidad de poder descifrar estrategias comunicacionales. Son ellos los indicados en la formación de una ciudadanía crítica en el consumo de medios. De esta manera, los receptores podrán analizar y reportar las expresiones y manifestaciones racistas." (MCP, 2014: 12)

Para Yaku Pérez (2016), presidente de la ECUARUNARI el pilar fundamental que debe regir los lineamientos comunicacionales de su organización son claros: "Una línea absolutamente crítica, diferente, que profundiza la realización, en armonía con la radicalidad de la información, es decir, procurar que la comunicación sea realmente objetiva, veraz e irreverente desde lo que hacemos. Creemos firmemente que la comunicación cuando viene desde los pueblos, cuando ellos tienen voz propia, ahí la comunicación se vuelve subversiva, frente al poder, frente al autoritarismo y frente al sistema. Y allí, buscamos hacer conciencia para enfrentar y combatir a un sistema capitalista, extractivista, patriarcal y racista que tanto daño nos ha hecho. Entonces, tenemos que apostar por una comunicación que descolonice las epistemes, los sentimientos, y todas las formas que se tienen sobre la concepción del mundo, y solamente después, caminar hacia una autentica cosmovisión y vivencias de los pueblos”.

El debate por una comunicación alternativa a un sistema mediático hegemónico, y a un sistema patriarcal, racista, capitalista y extractivista nos lleva a construir y proponer para la ECUARUNARI dirigir los medios de comunicación en base al proyecto de utilizar recursos online gratuitos, fáciles de usar y de buen alcance al público, tales como:

Mailing, blogs, perfiles en redes sociales como Facebook, Twitter, Linked In en las cuales se podría compartir comunicados, convocatorias, boletines de prensa, etc. complementadas con imágenes o video apoyándose en plataformas como Youtube, Flickr, Instagram, Uestream, publicar revistas interactivas o libros en a Issuu, una herramienta, fácil de usar y agradable para los lectores.

Con la conformación de canales oficiales la Confederación de Pueblos de la Nacionalidad Kichwa del Ecuador - ECUARUNARI, se beneficia en su fortalecimiento comunicacional ya que si cuenta con medios de difusión, la información compartida responde al contexto propio acorde a sus principios, con una capacidad de respuesta inmediata.

\section{Conclusiones}

El movimiento indígena del Ecuador cuenta con una trayectoria de lucha que lo ha posicionado como referente de los movimientos sociales en el país, solamente posible a la lealtad y fidelidad a sus principios fundamentales, a la solidaridad con los demás 
sectores y a la unidad en la construcción de la plurinacionalidad y la interculturalidad de la nación.

Conforme avanzan las plataformas de lucha del movimiento indígena, la comunicación crece en importancia como una herramienta estratégica para los procesos de liberación de las sociedades, por lo cual cada organización deberá trabajar en la definición de una línea política clara para el manejo y articulación de su comunicación y su relación, con las bases y con otras organizaciones.

Si bien, el factor económico representa una de las principales dificultades para la continuidad del proyecto comunicacional de la Confederación de Pueblos de la Nacionalidad Kichwa del Ecuador (ECUARUNARI), es fundamental buscar alternativas que lo permitan, además de tener claro que no solamente basta con tener un espacio en la red o una página web, sino que es primordial planificar los contenidos para lograr un posicionamiento en la red.

Finalmente, concluir diciendo que los ecuatorianos tenemos derecho a una comunicación intercultural pero lo que prima es una violencia política y sistémica que se ha institucionalizado en el país. Es una violencia que si bien nos invisibiliza, no nos doblega. El compromiso de las organizaciones es por la defensa del territorio, en contra la minería, la explotación petrolera y maderera. Es una lucha constante por asegurar la armonía con la Madre Tierra.

\section{Lista de referencias}

Amado, A. (2011). Auditoria de comunicación: un método de análisis de las comunicaciones públicas. Editorial DIRCOM, 2011. Buenos Aires . Argentina.

Corrales, F. y Hernández, H. (2009). La comunicación alternativa en nuestros días: un acercamiento a los medios de la alternancia y la participación. Revista Electrónica en América Latina Especializada en Comunicación RAZÓN Y PALABRA disponible en: http://www.razonypalabra.org.mx/N/N7o/CORRALES-HERNANDEZREVISADO.pdf

ECUARUNARI. (1981). El Movimiento campesino Indígena "ECUARUNARI".Documento elaborado para el Primer encuentro Nacional de Trabajadores del arte y la cultura del Ecuador "QUIPUCAMAYUC". Quito

Eizaguirre, M., Urrutia, C., y Askunze, C. (2004). La sistematización, una nueva mirada a nuestras prácticas. Instituto de estudios sobre desarrollo y cooperación internacional. Guía para la sistematización de experiencias de transformación social. Alboan, Instituto de Derechos Humanos Pedro Arrupe y Hegoa. Bilbao. 
Francois, F. (1977). El lenguaje: La comunicación. Editorial: Nueva Visión. Serie Tratado del Lenguaje No. 1, 184 pgs., Buenos Aires.

Gómez, R. (2008) Indígenas urbanos en Quito: el proceso de etnogénesis del pueblo Kitukara, en García, F. (Compilador). Identidades, etnicidad y racismo en América latina. FLACSO, Ministerio de Cultura del Ecuador. Quito.

Hobsbawn, E. (1992). Naciones y Nacionalismo desde 1780, 2.da edición. Colección: Divulgación. Editorial Booket.

Macas, L. (2002). La lucha del movimiento indígena en el Ecuador. Boletín ICCI-ARI Rimay, 4 (37). Quito.

MCP. (2014). Comunicación que NO discrimina: Guía para comunicadores. Ministerio de Cultura de Perú. Lima.

Moctezuma, N. (2011). La universidad intercultural de las nacionalidades y pueblos indígenas de Ecuador, Amawtay Wasi, En el contexto del movimiento indígena. (Tesis). Universidad Nacional Autónoma de México - UNAM. Facultad de Ciencias Políticas y Sociales. Centro de Estudios Sociológicos. México.

Pita S., y Pértegas, S. (2002). Investigación cuantitativa y cualitativa. (9), 76-78. Coruña (España). Recuperado en: https://www.fisterra.com/mbe/investiga/cuanti_cuali/cuanti_cuali.asp

RUIICAY. (2014). Cultivo y Crianza de Sabidurías y Conocimientos (CCRISAC). Red de Universidades Indígenas Interculturales y Comunitarias de Abya Yala.

Tavares, E. (1998). En torno al periodismo de la comunidad. Revista Latinoamericana de Comunicación Chasqui No. 61. CIESPAL. Quito 